19 Revue d'histoire du XIXe siècle

Société d'histoire de la révolution de 1848 et des

révolutions du XIXe siècle

$57 \mid 2018$

Libido sciendi

\title{
Hervé GUILLEMAIN et Nathalie RICHARD (eds), « The Frontiers of Amateur Science (18th-20th Century) »
}

Jean-Luc Chappey

URL : https://journals.openedition.org/rh19/6133

DOl : $10.4000 /$ rh19.6133

ISSN : $1777-5329$

Éditeur

La Société de 1848

Édition imprimée

Date de publication : 26 décembre 2018

Pagination : 197-200

ISSN : 1265-1354

Référence électronique

Jean-Luc Chappey, "Hervé guillemain et Nathalie richaRd (eds), «The Frontiers of Amateur Science

(18th-20th Century) » », Revue d'histoire du XIXe siècle [En ligne], 57 | 2018, mis en ligne le 26 décembre 2018, consulté le 20 janvier 2022. URL : http://journals.openedition.org/rh19/6133 ; DOI : https:// doi.org/10.4000/rh19.6133

Ce document a été généré automatiquement le 20 janvier 2022.

Tous droits réservés 


\title{
Hervé GUILLEMAIN et Nathalie RICHARD (eds), " The Frontiers of Amateur Science (18th-20th Century)»
}

\author{
Jean-Luc Chappey
}

\section{RÉFÉRENCE}

Hervé GUILLEMAIN et Nathalie RICHARD (eds), « The Frontiers of Amateur Science (18th-20th Century) », dossier de la revue Gesnerus. Swiss Journal of the History of Medicine and Sciences, vol. 73, $\mathrm{n}^{\circ} 2,2016,392$ p., 55

1 Si la notion d'amateurs a servi pendant longtemps à distinguer tous les acteurs, institutions, productions ou pratiques qui ne relèvent pas de la sphère professionnelle, elle mérite aujourd'hui d'être largement interrogée. C'est ce que propose la série de contributions réunies dans le numéro de Gesnerus autour du thème "The Frontiers of Science (18th-20th Century) ». Dans une introduction particulièrement lumineuse sur le plan historiographique et méthodologique («Towards a Contemporary Historiography of Amateurs in Science »), Nathalie Richard et Hervé Guillemain, coordinateurs du numéro, justifient la nécessité d'interroger en profondeur ce monde des amateurs qui ne saurait être considéré comme homogène et que l'on ne saurait réduire aux simples figures de vaincus ou de marginaux. Forgée au XIX ${ }^{\mathrm{e}}$ siècle (en particulier par le biais des "sociétés d'amateurs»), cette notion d'amateurs s'est progressivement imposée comme structurante dans l'historiographie des sciences : elle tend à regrouper tous les acteurs, institutions et productions qui n'entreraient pas - ou auraient été exclus - des contours du monde des savants professionnels. En un mot, les amateurs - dont Flaubert dessine les traits avec Bouvard et Pécuchet - renverraient à cette cohorte hétérogène de doux rêveurs, collectionneurs et curieux qui, à l'écart ou parfois contre le monde des sciences, chercheraient à conquérir, en dehors des procédures de reconnaissance légitime, une réputation. Depuis plusieurs décennies, ce schéma a été mis en question: l'histoire des sociabilité des élites nobiliaires ou bourgeoises, l'histoire culturelle à 
travers l'intérêt porté aux médiateurs et intermédiaires, ou l'histoire des pratiques et des espaces savants, comme les apports des gender studies, ont permis de nuancer cette lecture en révélant la vitalité et la complexité de ce monde savant dont les membres ont, entre l'époque moderne et contemporaine, largement participé aux dynamiques de production et de diffusion des savoirs légitimes. Comme en témoignent aujourd'hui les nombreuses interventions d'acteurs (comme les lanceurs d'alerte), groupes ou associations dans les luttes qui rythment les débats autour du réchauffement climatique, des questions de santé ou de la politique nucléaire, les frontières entre les professionnels, les experts, et les amateurs méritent d'être largement mises en cause tant il apparaît que la production et les modalités de diffusion de savoirs légitimes ne sauraient être monopolisés par un groupe restreint de spécialistes des savoirs consacrés. De même, l'encyclopédie gratuite en ligne, Wikipédia, valorise désormais l'intervention de rédacteurs, souvent anonymes et de plus en plus nombreux, qui alimentent les notices sans passer par le contrôle des autorités académiques. S'il est possible de distinguer un professionnel d'un amateur (par les formes de reconnaissance institutionnelle ou de financement), il n'en reste pas moins vrai qu'il est impossible d'affirmer que l'un serait, par nature, moins légitime que l'autre. C'est ce que montrent les différentes études réunies dans ce volume, qui s'attachent à étudier précisément les trajectoires individuelles d'acteurs considérés comme des amateurs et à questionner certaines pratiques qui, ne relevant pas des institutions dominantes, ont pourtant joué un rôle essentiel dans l'histoire des sciences. À travers l'étude de l'itinéraire de deux entomologistes, Charles Janet, ingénieur de province peu connu mais très actif au sein de La Société entomologique de France, et Eugène Louis Bouvier, académicien et titulaire de la chaire d'entomologie au Muséum national d'histoire naturelle, Loïc Casson montre ainsi qu'entre les deux les démarcations sont "subtiles ». Certains domaines de savoir (nécessitant observations, collectes...), tels des "niches», apparaissent comme plus favorables aux amateurs : archéologie, botanique, astronomie ou science de l'esprit. Néanmoins, là encore, il convient d'être prudent car le partage entre savants sédentaires et amateurs collecteurs n'est, par exemple, pas clairement établi, alors que les savants consacrés (dans les études ethnologiques notamment) continuent de pratiquer l'enquête de terrain. Anne-Gaëlle Weber s'interroge de son côté sur les enjeux de la distanciation entre les productions scientifiques et les productions littéraires, les amateurs se voyant progressivement ravalés au statut d'hommes de lettres. Contrairement à l'idée reçue d'un conflit entre le "style sévère " du savant et le "style » de l'écrivain, les frontières peuvent rester poreuses entre les tenants de l'écriture scientifique et littéraire. En étudiant les trajectoires des ornithologues professionnels des années 1800 , elle montre comment ces derniers ont justement construit les contours épistémologiques de la description des oiseaux en jouant sur les deux types d'écriture, nomenclatures et descriptions esthétiques.

2 Il s'agit dès lors de questionner les conditions et les modalités de construction de la figure de l'amateur qui, produit d'un processus singulier qu'il convient d'étudier, se caractérise par sa complexité et sa position toujours susceptible de changement. Contrairement à l'historiographie traditionnelle qui voudrait faire de l'amateur une figure immuable et envers de celle du professionnel, le statut de l'amateur, dépendant de jeux de pouvoir ou de stratégies particulières, reste temporaire et fragile. La réputation de l'amateur ou sa carrière ne sont pas restreintes à une échelle locale, et l'amateur peut même conquérir un statut de professionnel et le perdre. Jacqueline Carroy rappelle ainsi le statut de «l'amateur volontaire», tel Marcel Sembat, pour qui 
la psychologie est un hobby et qui pratique cette activité dans la sphère privée. Dans son étude de la trajectoire d'Anne Berman (1889-1979), Rémy Amouroux montre comment cette pharmacienne de profession, propriétaire de son officine, investit progressivement le champ de la psychanalyse: secrétaire personnelle de Marie Bonaparte de 1933 à 1962, elle bénéficie d'une reconnaissance au sein du monde savant, parallèlement au rôle essentiel qu'elle joue au sein de l'association (Soroptimist) de défense des intérêts des femmes. Néanmoins, elle se heurte à des oppositions qui rappellent que la frontière entre professionnels et amateurs est toujours affaire de rapports de force: si elle s'impose progressivement comme la traductrice officielle des œuvres de Freud, elle se heurte à des contestations croissantes émanant de concurrents qui, pour la marginaliser, insistent sur sa position subalterne au sein du monde des psychanalystes et lui dénient toute forme de légitimité scientifique. Dans la même perspective, Claire Gantet montre comment une tentative d'institutionnalisation de la psychologie a été menée dans l'Allemagne du dernier tiers du XVIII ${ }^{\mathrm{e}}$ siècle, par le biais d'un périodique (le Magazin zur Erharungseelenkunde) dont l'objectif était de recueillir les récits individuels afin de mieux normaliser la médecine morale. S'appuyant sur des acteurs et des réseaux qui se trouvent en dehors des universités, cette tentative suscite une contre-offensive au moment où, dans ces institutions, les professeurs cherchent à faire valoir leur autorité et leur contrôle sur les différents domaines de savoirs : à partir de 1790, l'entreprise fait ainsi l'objet de critiques qui permettent à des professeurs de se réapproprier les règles et les contours de la psychologie et de se poser comme les seuls intervenants légitimes en matière de "science de l'âme ». Volny Fages met en lumière les règles et les codes à partir desquels se joue la réputation savante dans la deuxième moitié du XIX ${ }^{e}$ siècle: la marginalisation de l'autodidacte Chacornac de son poste d'astronome titulaire de l'observatoire de Paris, sa relégation progressive en dépit de ses efforts pour rester dans la course, font apparaitre les contraintes (en termes de dispositions, de savoir-être, et de compétences culturelles) qui pèsent désormais sur le monde des sciences, contraintes d'autant plus difficiles à dépasser qu'elles sont souvent tacites.

3 Les différents auteurs confirment ainsi chacun la valeur heuristique de la notion d'amateurs qui permet non seulement de faire apparaître des acteurs souvent méconnus ou rendus invisibles, mais aussi d'interroger des processus d'organisation du monde savant qui restent oubliés lorsqu'on se contente d'étudier les institutions et les acteurs consacrés. 